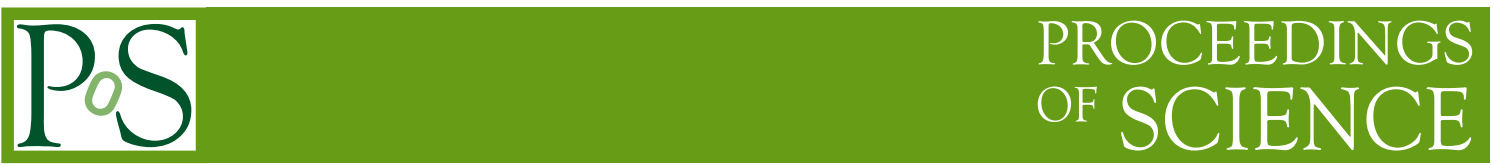

\title{
Modern amplitude method studies of effective field theories
}

\author{
Karol Kampf* \\ Institute of Particle and Nuclear Physics, Charles University, Prague, Czech Republic \\ E-mail: karol.kampf@mff.cuni.cz
}

\begin{abstract}
Description of low-energy interactions of Goldstone particles of spontaneously broken symmetries is traditionally done using the most general action based on the underlying symmetry. All such possible terms are taken up to a given order in the low energy expansion. The explicitly constructed Lagrangian can be then used for calculating given scattering amplitudes. However this process is not very efficient as can be demonstrated already at the tree-level for relatively small number of external particles. The modern amplitude methods offer different approach where it is possible to avoid redundancy stemming from the standard Feynman diagram calculations. We will explicitly show this in the simplest case of multi-pion scatterings. The methods represent not only an efficient way of calculating scattering amplitudes but can be employed in theoretical studies of their interesting properties. This allows among other things to classify the effective models for scalar bosons. A brief discussion of higher spin will be also given.
\end{abstract}

The 9th International workshop on Chiral Dynamics

17-21 September 2018

Durham, NC, USA

${ }^{*}$ Speaker. 


\section{Introduction}

Chiral perturbation theory (ChPT) represents one of the most important examples of effective field theories in particle physics. It is important both from a theoretical research and practical perspective. It is an example of the non-trivially realized spontaneous symmetry breakdown via the coset construction of $G / H$. It is also phenomenologically rich theory as can be verified in nature by studying the low-energy properties of the corresponding Goldstone bosons - pseudoscalar mesons. More specifically we talk about the three-flavour case if the group pattern is $S U(3)_{L} \times S U(3)_{R} \rightarrow$ $S U(3)_{V}$ or the two-flavour case for the symmetry breaking $S U(2)_{L} \times S U(2)_{R} \rightarrow S U(2)_{V}$. In the three-flavour case corresponding theory describes the interactions of pions, kaons and eta (eight particles in total) and in the two-flavour case it focuses only on pions (three in total: $\pi^{ \pm}$and $\pi^{0}$ ). It was first developed at the tree-level [1] and later up to NNNLO (or equivalently up to $O\left(p^{8}\right)$, where $p$ stands for a generic momentum) in [2]. The connection of many low-energy constants with experimental measurements is one of the main phenomenological task in this field. There are also many open theoretical questions - for example resummation of leading logarithms (see e.g. [3]) or role of resonances in the low energy constant saturation (c.f. [4]). In this work, however, we will return back to 'roots': at the tree level and try to look at the problem from a completely different perspective.

\section{Role model: gluon amplitudes}

Main motivation can be seen in calculating the gluon amplitudes. At tree-level focusing only on gluons in the final state we can limit ourselves only to pure gluon vertices and we can forget about quarks as they enter only via loops. With this simplification it seems easy to proceed there are two basic interaction vertices: three-gluon and four-gluon one. If we want to calculate a process with $n$-external gluon legs we must sum together all possible combinations of these two main building blocks connected with gluon propagators. For the four-point scattering we have in total four diagrams to evaluate. Those diagrams (depicted in Fig. 1) are relatively easy to calculate, however, for the higher orders the number of diagrams exponentially rises. For example for $g+g \rightarrow$
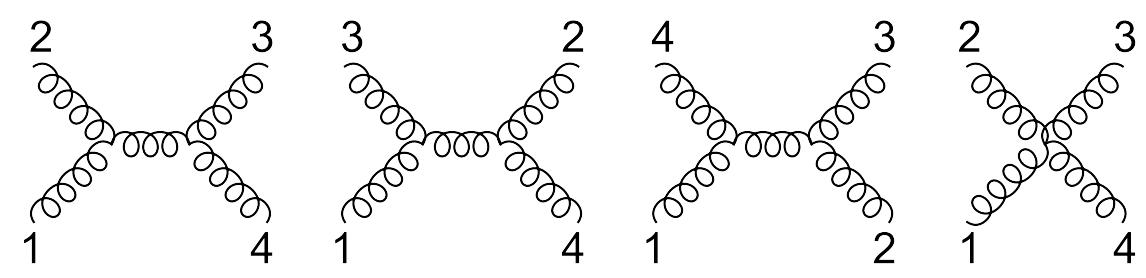

Figure 1: All tree-level Feynman diagrams for the four-gluon scattering

$g+g+g$ it is already 25 diagrams as one can still easily verify, whereas for a 6pt scattering it is 220 diagrams. It is instructive but also important for our further discussion to actually calculate the number of diagrams systematically. One can easily write a recursive program to generate Feynman diagrams or use a trick via the simplified equations of motion [5] to get a recursive formula. The summary for different theories is given in Tab. 1. From this exponential growth it is clear that 


\begin{tabular}{|c|c|c|c|c|c|c|c|c|}
\hline $\mathrm{n}$ & 3 & 4 & 5 & 6 & 7 & 8 & 9 & 10 \\
\hline $3 \mathrm{pt}+4 \mathrm{pt}$ & 1 & 4 & 25 & 220 & 2485 & 34300 & 559405 & 10525900 \\
\hline even-pt & 0 & 1 & 0 & 11 & 0 & 337 & 0 & 20267 \\
\hline odd-pt & 1 & 3 & 16 & 120 & 1156 & 13608 & 189316 & 3039060 \\
\hline all-pt & 1 & 4 & 26 & 236 & 2752 & 39208 & 660032 & 12818912 \\
\hline
\end{tabular}

Table 1: Number of Feynman diagrams for different theories: Young-Mills theories with trilinear and quadrilinear couplings (e.g. gluon scattering), effective field theories with only the even number of legs (ChPT for pions), with only the odd number of legs (not very realistic theories - e.g. galileon theories with odd vertices in general dimension) and finally with any number of legs for interaction vertices (corresponds e.g. to gravity).

"standard" evaluation of scattering amplitudes via the Feynman diagram perturbation is not very useful in situations that involve higher number of particles. We can ask for alternative resummation with preferably much lower number of terms at individual orders.

One important step in this direction, applicable in the case of gluons, is realising that it is possible to define the so-called stripped amplitudes by

$$
\mathscr{A}_{n}^{\text {full }}=\sum_{\sigma / Z_{n}} A_{n}\left(p_{\sigma(1)} \ldots p_{\sigma(n)}\right) \operatorname{Tr}\left(T^{\sigma(1)} \ldots T^{\sigma(n)}\right),
$$

where the sum is over all non-cyclic permutations. Derivation is based on the colour algebra $\left(T^{i}\right.$ are its generators) and can be verified for particles transforming in the adjoint representation of the gauge group. One important thing about the colour-ordered amplitude is that it contains all physically relevant information, which is a trivial consequence of the fact the form of the single term $A_{n}$ is needed to obtain the complete amplitude in (2.1). It is not then surprising that the stripped amplitude is gauge invariant. Let us stress again that the above relation is true only at the tree level and for the loop-order diagrams one should consider modification with multiple traces in such generalized formula. But back to the ordered amplitudes. Already this considerably simplifies the calculation. First of all a propagator in the stripped amplitude can be obtained only with consecutive momenta, i.e. it is of the form:

$$
P_{i j}^{2}=\left(p_{i}+p_{i+1}+\ldots+p_{j}\right)^{2}, \quad \text { for } i<j .
$$

We can then come with a nice geometrical interpretation, first mentioned in [6], where every stripped diagram with ordered vertices for the $n$-pt scattering can be represented by a convex regular $n$-gon (square, pentagon, etc). Propagators are represented by non-crossing diagonals. This is an amusing connection, however, so far without much of impact in this field. One, though, application is again connected with counting of the ordered diagrams. We can repeat a similar calculations as for Tab. 1. The results are summarized in Tab. 2. The numbers are interesting on their own - for example the last line (the all-pt) represents the so-called super-Catalan numbers. At this moment it is, however, important that they clearly show that our task to simplify calculation of the scattering amplitudes is successful. Nevertheless the grow is still exponential and we must ask for some more radical approach. One such is the so called BCFW recursion [7]. Let us summarize here its basic characteristic. 


\begin{tabular}{|c|c|c|c|c|c|c|c|c|}
\hline $\mathrm{n}$ & 3 & 4 & 5 & 6 & 7 & 8 & 9 & 10 \\
\hline $3 \mathrm{pt}+4 \mathrm{pt}$ & 1 & 3 & 10 & 38 & 154 & 654 & 2871 & 12925 \\
\hline even-pt & 0 & 1 & 0 & 4 & 0 & 21 & 0 & 126 \\
\hline odd-pt & 1 & 2 & 6 & 20 & 71 & 264 & 1015 & 4002 \\
\hline all-pt & 1 & 3 & 11 & 45 & 197 & 903 & 4279 & 20793 \\
\hline
\end{tabular}

Table 2: Number of ordered diagrams for different theories defined in Tab. 1.

We start with a simple observation. Taking any existing propagator which must be of the form (2.2) and set it to zero. Amplitude then factorizes

$$
\lim _{P_{1 j}^{2} \rightarrow 0} M(1,2, \ldots n)=\sum_{h_{l}} M_{L}(1,2 \ldots j, l) \cdot \frac{1}{P_{1 j}^{2}} \cdot M_{R}(l, j+1, \ldots n) .
$$

In order to reconstruct the amplitude from its poles in complex plane we will make use of the following trick. We will shift some two external momenta (usually the adjacent ones)

$$
p_{i} \rightarrow p_{i}+z q, \quad p_{j} \rightarrow p_{j}-z q,
$$

so trivially the momentum conservation will not be altered and the shifted $p_{i}$ and $p_{j}$ remain onshell, i.e.

$$
q^{2}=q \cdot p_{i}=q \cdot p_{j}=0 .
$$

The amplitude thus becomes a meromorphic function $A(z)$ of the complex parameter $z$. To reproduce back the original function one takes $z=0$. It is important to notice that we can have only simple poles coming from propagators $P_{a b}(z)$. The Cauchy's theorem now implies

$$
\frac{1}{2 \pi \mathrm{i}} \int \frac{d z}{z} A(z)=A(0)+\sum_{k} \frac{\operatorname{Res}\left(A, z_{k}\right)}{z_{k}} .
$$

Assuming that $A(z)$ vanishes for $z \rightarrow \infty$ we get for the original amplitude (taking trivially $z \rightarrow 0$ )

$$
A=A(0)=-\sum_{k} \frac{\operatorname{Res}\left(A, z_{k}\right)}{z_{k}} .
$$

The propagator $P_{a b}^{2}(z)=0$ if one and only one of the two shifted momenta is in $(a, a+1, \ldots, b)$. Then the solution is

$$
z_{a b}=-\frac{P_{a b}^{2}}{2\left(q \cdot P_{a b}\right)}
$$

and for the allowed helicities it factorizes into two subamplitudes

$$
\operatorname{Res}\left(A, z_{a b}\right)=\sum_{s} A_{L}^{-s}\left(z_{a b}\right) \frac{1}{2\left(q \cdot P_{a b}\right)} A_{R}^{s}\left(z_{a b}\right) .
$$

Using Cauchy's formula (2.6), we have finally

$$
A=\sum_{k, s} A_{L}^{-s_{k}}\left(z_{k}\right) \frac{1}{P_{k}^{2}} A_{R}^{s_{k}}\left(z_{k}\right) .
$$

It is clear that number of terms is now linear, at order $n$ equals maximally to $2 \times(n-3)$. Here 2 represents two possible helicity configurations in the above sum. 


\section{BCFW for effective field theories}

Important assumption above that $A(z)$ vanishes for $z \rightarrow \infty$ is not necessary true for a general case. In such situations we have to include a boundary term in the Cauchy theorem. We can demonstrate it on the example of scalar QED, given by the Lagrangian

$$
\mathscr{L}=-\frac{1}{4} F_{\mu \nu} F^{\mu \nu}-\left|D_{\mu} \phi\right|^{2}-\frac{1}{4} \lambda|\phi|^{4} .
$$

Due to the power-counting the boundary term is proportional to

$$
B \sim 2 e^{2}-\lambda
$$

If we take blindly the BCFW formula and reconstruct some $n$-point amplitude based on the threepoint vertex $(\sim e)$ we get a result which is independent of $\lambda$ in (3.1). What does this result represent? The answer is given in (3.2): the boundary term must be zero and thus it corresponds to a theory with $\lambda=2 e^{2}$. We can put it other way round. We are looking for some principle which would connect the ' $\lambda$-piece' with other part of $\mathscr{L}$. In this case it is a supersymmetry. For a general effective field theory, schematically written as

$$
\mathscr{L}=\frac{1}{2}(\partial \phi)^{2}+\lambda_{4}(\partial \phi)^{4}+\lambda_{6}(\partial \phi)^{6}+\ldots
$$

we have infinite towers of such $\lambda$ terms. Calculating for example the 6pt scattering, where Feynman diagrams are
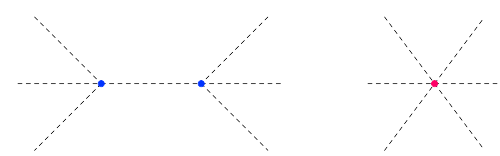

and the corresponding amplitude is (schematically):

$$
\mathscr{A}_{6}=\sum_{I=\text { poles }} \lambda_{4}^{2} \frac{\cdots}{P_{I}}+\lambda_{6}(\ldots) .
$$

Now our task is to find a way how to connect the contact term (represented by $\lambda_{6}$ ) with the factorization channels $\left(\sim \lambda_{4}^{2}\right)$. This question cannot be answered in general for all effective field theories. We will thus start our study with the most important example - the non-linear sigma model (NLSM). The Lagrangian is given by

$$
\mathscr{L}=\frac{F^{2}}{4}\left\langle\partial_{\mu} U \partial^{\mu} U^{-1}\right\rangle
$$

where

$$
U=\exp \left(\sqrt{2} \frac{i}{F} \phi\right)
$$

with $\phi=\phi^{i} t^{i}$ and $t^{i}$ are generators of the chiral group (in practise $S U(2)$ or $S U(3)$ ).

The answer about some defining property is well known for this theory and is called Adler zero condition. More specifically, being in the massless limit we have no ambiguity how to take any external momenta to zero and keeping the on-shell condition:

$$
p_{i} \rightarrow 0, \quad \text { and } \quad p_{i}^{2}=0
$$


Then the amplitude has to vanish

$$
\left.\mathscr{A}_{n}\left(p_{1}, \ldots, p_{n}\right)\right|_{p_{i} \rightarrow 0}=0 .
$$

Before studying the consequences of this condition, let us first try to repeat what was important in the gluon case: the stripping of the amplitudes. We will focus only on the group $S U\left(N_{f}\right)$. Using the properties of the structure constants we can prove that the interaction vertices can be ordered. Employing then the completeness relations we can as well build the ordered amplitude using the stripped vertices exactly as in the gluon case. The amplitude has then a simple group structure

$$
\mathscr{A}_{n}^{a_{1} \ldots a_{n}}=\sum_{\sigma / Z_{n}} A_{n}\left(p_{\sigma(1)} \ldots p_{\sigma(n)}\right) \operatorname{Tr}\left(t^{a_{\sigma}(1)} \ldots t^{a_{\sigma}(n)}\right)
$$

where $a_{i}$ labels the flavour of an $i$-th particle. Due to the construction of both vertices and striped amplitudes it can be easily proved their uniqueness. We can thus transform a general property of the amplitude, in this case the Adler zero, onto the stripped amplitude, similarly as was the gauge symmetry in the gluon case. Already now with possession only of two basic things: possibility of stripping and the Adler zero we can easily reconstruct recursively in principle any $n$-point NLSM amplitude just from the knowledge of the $4 \mathrm{pt} \mathrm{vertex.} \mathrm{For} \mathrm{example} \mathrm{the} 6 \mathrm{pt}$ amplitude is then given by a compact form using a cycling property

$$
4 F^{4} A_{6}(1,2,3,4,5,6)=-\frac{1}{2} \frac{\left(s_{12}+s_{23}\right)\left(s_{45}+s_{56}\right)}{\left(p_{1}+p_{2}+p_{3}\right)^{2}}+s_{12}+\mathrm{cycl},
$$

where we have introduced standard Mandelstam variables: $s_{i j}=\left(p_{i}+p_{j}\right)^{2}$. We can continue and easily calculate 8pt amplitude (see e.g. [8]), which means to deal with in total 21 diagrams (see Tab.2). However, some of them are only simple cyclic permutation and in fact we have to deal only with four topologies. These are:
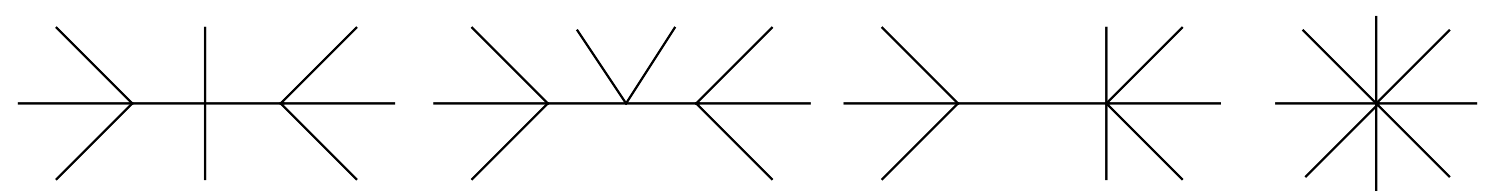

Including the cyclic ones we have in total: $4+8+8+1=21$ diagrams which agrees with the total number. Note important difference in normal topologies and the cyclic one: for the normal Feynman diagrams we would not distinguish between the first and the second topology.

Similarly we can continue with a ten-point scattering: 126 diagrams (however spanned on 16 topologies). The 12 point case with 818 diagrams would be still possible to calculate using conventional method but clearly again we are basically back to the original problem - number of diagrams grows rapidly. In the gluon case we have continued with the BCFW recursion relation. It is natural to seek for the alternative as well for the effective field theories. Generally speaking the main obstacle of using analytic properties here is hidden in the high energy properties of amplitude at $z \rightarrow \infty$. Let us try to solve this problem for a general effective field theory.

One important piece of information which is not automatically employed in the BCFW construction is amplitudes' low energy behaviour. Typically, amplitudes in effective field theories 
vanish in the limit $p \rightarrow 0$ for one external momentum as we have learnt above for NLSM. We might thus define a classification of theories according to the degree of their soft behaviour using a parameter $\sigma$

$$
A_{n} \sim p^{\sigma} \text { for } p \rightarrow 0 .
$$

$\sigma$ must be an integer (it was equals to 1 for NLSM). It is clear that if $\sigma$ is positive we get a nontrivial constraint which we can use to fix the amplitude. One important ingredient is still missing in order to fully exploit (3.9) and this is the momentum shift. This is not accomplished by the BCFW shift defined in (2.4) as it does not probe the soft limits of external legs. We will define instead

$$
p_{i} \rightarrow p_{i}\left(1-z a_{i}\right)
$$

and to be self-consistent we will have to demand

$$
\sum_{i=1}^{n} a_{i} p_{i}=0
$$

otherwise we would lose the momentum conservation. It is clear that if $a_{i}$ are all equal we have simple rescaling which cannot bring anything new. It is an easy algebraic task to demand other than this trivial solution. We must have linearly dependent momenta in order to avoid non-zero determinant. This can happen for $n>D+1$ when we can find solutions with distinct $a_{i}$ s for general $p_{i}$ configuration. One nice property of the chosen form for the shift is that

$$
A_{n}(z) \sim\left(1-z a_{i}\right)^{\sigma} \text { for } z \rightarrow 1 / a_{i} .
$$

Now comes a crucial point: instead of applying directly the Cauchy's formula on the amplitude we will try to reconstruct $A(n) / F(z)$ with

$$
F_{n}(z)=\prod_{i=1}^{n}\left(1-a_{i} z\right)^{\sigma}
$$

We will assume a vanishing boundary term at $z \rightarrow \infty$, which is more probable as the term $F_{n}(z)$ improves the large $z$ behaviour. The Cauchy formula is then

$$
\oint \frac{d z}{z} \frac{A_{n}(z)}{F_{n}(z)}=0 .
$$

Note that the poles introduced by $F_{n}(z)$ are cancelled by zeroes of the amplitude. Then in analogy with BCFW, the amplitude is

$$
A_{n}(0)=-\sum_{I} \operatorname{Res}_{z=z_{I \pm}}\left(\frac{A_{n}(z)}{z F_{n}(z)}\right),
$$

where $I$ again labels factorization channels. In contrast with BCFW, the each factorization channel in $P_{I}(z)$ yields two poles $z_{I \pm}$ corresponding to the roots of

$$
P_{I}^{2}+2 P_{I} \cdot Q_{I} z+Q_{I}^{2} z^{2}=0
$$

where $P_{I}(z)=P_{I}+z Q_{I}$ and where

$$
P_{I}=\sum_{i \in I} p_{i} \quad \text { and } \quad Q_{I}=-\sum_{i \in I} a_{i} p_{i}
$$


It is clear that each residue is a product of lower-point amplitudes which can be rearranged into a new recursion relation [9],

$$
A_{n}(0)=\sum_{I} \frac{1}{P_{I}^{2}} \frac{A_{n_{I}}\left(z_{I-}\right) A_{\bar{n}_{I}}\left(z_{I-}\right)}{\left(1-z_{I-} / z_{I+}\right) F_{n}\left(z_{I-}\right)}+\left(z_{I+} \leftrightarrow z_{I-}\right) .
$$

This concludes our task to reconstruct theory using a generalization of the BCFW formula using all available information of the soft momentum behaviour.

\section{Classification of effective field theories}

In the previous section we have improved the behaviour of analytically continued amplitude at infinity. However, we have not discussed if this improvement is sufficient and if in fact we can use the Cauchy theorem in the form (3.13). The formula was derived for a general effective theory but to answer this question we have to return to some actual model. We can study directly NLSM and see if it fulfils requirements. Instead we choose to discuss it from a different perspective and more generally. We will ask what theories are reconstructible using the above defined modified BCFW recursion. As we will see NLSM will pop out naturally also using this way.

We start with a definition of another natural parameter which will roughly-speaking counts the homogeneity. Any interaction term can be written in general as

$$
\mathscr{L}_{m, n}=\partial^{m} \phi^{n}
$$

which is a schematic representation of all terms with $m$ derivatives and $n$ fields. Of course the full Lagrangian is a sum of combinations of such terms. We will define a natural parameter $\rho$

$$
\rho=\frac{m-2}{n-2},
$$

which will enable to pick among different $\mathscr{L}_{m, n}$ those that have the same powercounting and can "communicate" together. One can easily verify this using the simplest non-trivial example of a 6pt scattering. It must be governed by $\mathscr{L}=\partial^{m} \phi^{4}+\partial^{\tilde{m}} \phi^{6}$. In order the propagator and contact diagrams are of the same order we must have $\tilde{m}=2 m-2$. This also means that $\rho=\tilde{\rho}$.

Now having in possession two parameters: $\sigma$ and $\rho$ we can ask what are the theories behind them. In the following we will focus only on $d=4$ dimension (for a more general discussion we refer to [10]). First we have to stress what are the interesting theories we are looking for. It is clear that having many derivatives in the interaction terms it is trivial to fulfil the low- $\sigma$ soft behaviour. We will state it more rigorously in the following. We will be interested in the non-trivial cases for $\mathscr{L}_{m, n}$ with

$$
m<\sigma n
$$

which can be easily rewritten as

$$
\sigma>\frac{(n-2) \rho+2}{n} .
$$

For our further discussion it is also crucial to realize that this naive threshold is also a threshold of the on-shell constructibility. In fact under the rescaling shift all momenta scale as $z$ for large $z$ and 
for the amplitude with $n$ particles and $m$ powers of momenta trivially

$$
A_{n}(z) \sim z^{m}, \quad F_{n}(z) \sim n \sigma \quad \rightarrow \quad \frac{A_{n}(z)}{F_{n}(z)} \sim z^{m-n \sigma}
$$

Demanding now at least $1 / z$ behaviour we ended up with exactly same condition (4.3). This simple statement has important consequences. In systematic searching for possible interesting theories we need only to enumerate the lowest- $n$ pt non-trivial scattering amplitudes. Such theories will be then valid for all $n$ thanks to the modified BCFW. Let us briefly summarize these non-trivial theories.

4.1 $\rho=0, \sigma=1$

Here and in the following we will focus only on two possible classes: single scalar case and multiple scalars with flavour-ordering. Schematically we are studying

$$
\mathscr{L}=\frac{1}{2}(\partial \phi)^{2}+\sum_{i} \lambda_{4}^{i}\left(\partial^{2} \phi^{4}\right)+\sum_{i} \lambda_{6}^{i}\left(\partial^{2} \phi^{6}\right)+\ldots
$$

for a single scalar and similar Lagrangian can be written for the multiple case. The outcome of the procedure is a free theory for a single scalar and NLSM for multi-flavours. It is amusing to notice that being in possession of the above modern tools (especially the modified BCFW) make these claims almost trivial and should be compare with similar studies done exactly 50 years ago [11].

4.2 $\rho=1, \sigma=2$

So far when discussing particular example of effective field theory we have used only NLSM. Now we have to get to something completely different which will have double soft limit. We can start with the lowest order non-trivial Lagrangian

$$
\mathscr{L}_{\text {int }}=c_{2}(\partial \phi \cdot \partial \phi)^{2}+c_{3}(\partial \phi \cdot \partial \phi)^{3}
$$

Calculating the six point amplitude and demanding the double soft limit leads to a condition $c_{3}=$ $4 c_{2}^{2}$. It means that there must be a non-trivial theory with a double soft behaviour up to all orders in number of external particles. What is actually this theory? We can either use the BCFW or focus on the symmetry responsible for this behaviour to find a condition for coefficients in $\mathscr{L}_{\text {int }}$ :

$$
2(n+1) c_{n+1}=(2 n-1) c_{n} .
$$

Setting the canonical kinetic term we get the full Lagrangian

$$
\mathscr{L}=-\sqrt{1-(\partial \phi . \partial \phi)},
$$

which is a well-known Dirac-Born-Infeld (DBI) action.

$4.3 \rho=2, \sigma=2$

Similarly to previous case we will arrive to a unique solution: the Galileon Lagrangian [12]

$$
\begin{aligned}
& \mathscr{L}=\sum_{n=1}^{d+1} d_{n} \phi \mathscr{L}_{n-1}^{\text {der }} \quad \text { with } \\
& \mathscr{L}_{n}^{\text {der }}=\varepsilon^{\mu_{1} \ldots \mu_{d}} \varepsilon^{v_{1} \ldots v_{d}} \prod_{i=1}^{n} \partial_{\mu_{i}} \partial_{v_{i}} \phi \prod_{j=n+1}^{d} \eta_{\mu_{j} v_{j}}=-(d-n) ! \operatorname{det}\left\{\partial^{v_{i}} \partial_{v_{j}} \phi\right\}_{i, j=1}^{n} .
\end{aligned}
$$

We can easily add also the multiflavour variants of this and similarly also for the DBI Lagrangian. For details we refer to [10]. 


\section{$4.4 \rho=2, \sigma=3$}

All theories were well known before we have suggested this procedure. However, there is one theory not known before starting this program. From the previous subsection we see that galileon theory has three parameters (in $d=4$ ). Due to dualities, however, only two are physically relevant [13]. We can ask if we can demand enhanced soft limit and the answer is yes. Indeed, there is a new theory, nowadays called special galileon. For details see [14]. The hidden symmetry responsible for this behaviour was discovered in [15].

\subsection{Spin=1}

The vector case is beyond the scope of this proceedings but it shows a potential of this method also for other types of particles. Here we will consider a massless degree of freedom which is described by the gauge invariant Abelian field strength tensor $F_{\mu \nu}=\partial_{\mu} A_{v}-\partial_{v} A_{\mu}$. All interactions can be expressed in terms of two building blocks

$$
F_{\mu v} F^{\mu v} \quad \text { and } \quad F_{\mu v} \tilde{F}^{\mu v}=\frac{1}{2} \varepsilon^{\mu v \rho \sigma} F_{\mu v} F_{\rho \sigma}
$$

The situation is now different from the scalar case. First of all there are two helicity states (+/-) of a massless vector. We can represent a momentum in a spinor helicity formalism and denote it as $\sim \lambda \tilde{\lambda}$. Now it is clear that there are more combinations how to go with a momentum to zero. After a thorough examination we have discovered one reasonable limit, called a multi chiral soft limit defined by keeping all helicity of one type soft:

$$
\lim _{\lambda_{+} \rightarrow \varepsilon \text { or } \tilde{\lambda}_{-} \rightarrow \varepsilon} A_{n}=O(\varepsilon) .
$$

By requiring this behavour one will get exactly one theory, known as the Born-Infeld (BI) model, which represents a nonlinear extension of Maxwell theory. Its Lagrangian is

$$
\mathscr{L}_{\mathrm{BI}}=1-\sqrt{-\operatorname{det}\left(\eta_{\mu v}+F_{\mu v}\right)} .
$$

For more details, mainly on possible recursion relations, proofs and extensions see [16].

\section{Summary}

We have briefly discussed how it is possible to apply the amplitude methods in the case of effective field theories. Among other things this program represents a continuation of activities that were already touched 50 years ago and represents in some sense their generalization. So far the systematic studies were focusing mainly on a massless single scalar or multiple scalars which allow for flavour ordering. First brief discussion on vector effective field theories was presented. Similarly there is a study focusing on fermions via supersymmetrization of galileon models [17].

\section{Acknowledgments}

This work was supported by the Czech Science Foundation (grant no. GAČR 18-17224S) and by the Ministry of Education (project LTAUSA17069). 


\section{References}

[1] S. Weinberg, Physica A 96 (1979) 327;

[2] J. Gasser and H. Leutwyler, Annals Phys. 158142 (1984); J. Gasser and H. Leutwyler, Nucl. Phys. B 250465 (1985); J. Bijnens, G. Colangelo and G. Ecker, JHEP 9902020 (1999) [hep-ph/9902437]; J. Bijnens, N. Hermansson-Truedsson and S. Wang, JHEP 1901, 102 (2019) [arXiv:1810.06834 [hep-ph]].

[3] J. Bijnens, K. Kampf and S. Lanz, Nucl. Phys. B 873, 137 (2013) [arXiv:1303.3125 [hep-ph]]; J. Bijnens, K. Kampf and S. Lanz, Nucl. Phys. B 860, 245 (2012) [arXiv:1201.2608 [hep-ph]].

[4] K. Kampf and B. Moussallam, Eur. Phys. J. C 47, 723 (2006) [hep-ph/0604125].

[5] C. Cheung,“TASI Lectures on Scattering Amplitudes,” arXiv:1708.03872 [hep-ph].

[6] L. Susskind and G. Frye, Phys. Rev. D 11682 (1970). doi:10.1103/PhysRevD.1.1682

[7] R. Britto, F. Cachazo and B. Feng, Nucl. Phys. B 715499 (2005) [hep-th/0412308]; R. Britto, F. Cachazo, B. Feng and E. Witten, Phys. Rev. Lett. 94181602 (2005) [hep-th/0501052].

[8] K. Kampf, J. Novotny and J. Trnka, Phys. Rev. D 87, no. 8, 081701 (2013) [arXiv:1212.5224 [hep-th]]; K. Kampf, J. Novotny and J. Trnka, JHEP 1305, 032 (2013) [arXiv:1304.3048 [hep-th]].

[9] C. Cheung, K. Kampf, J. Novotny, C. H. Shen and J. Trnka, Phys. Rev. Lett. 116, no. 4, 041601 (2016) [arXiv:1509.03309 [hep-th]].

[10] C. Cheung, K. Kampf, J. Novotny, C. H. Shen and J. Trnka, JHEP 1702020 (2017) [arXiv:1611.03137 [hep-th]].

[11] H. Osborn, Lett. Nuovo Cim. 2S1 717 (1969) [Lett. Nuovo Cim. 2717 (1969)], J. R. Ellis and B. Renner, Nucl. Phys. B 21205 (1970).

[12] G. W. Horndeski, Int. J. Theor. Phys. 10, 363 (1974);

A. Nicolis, R. Rattazzi and E. Trincherini, Phys. Rev. D 79, 064036 (2009) [arXiv:0811.2197 [hep-th]].

[13] C. de Rham, M. Fasiello and A. J. Tolley, Phys. Lett. B 73346 (2014) [arXiv:1308.2702 [hep-th]]; K. Kampf and J. Novotny, JHEP 1410006 (2014) [arXiv:1403.6813 [hep-th]].

[14] C. Cheung, K. Kampf, J. Novotny and J. Trnka, Phys. Rev. Lett. 114, no. 22, 221602 (2015) [arXiv:1412.4095 [hep-th]].

[15] K. Hinterbichler and A. Joyce, Phys. Rev. D 92, no. 2, 023503 (2015) [arXiv:1501.07600 [hep-th]].

[16] C. Cheung, K. Kampf, J. Novotny, C. H. Shen, J. Trnka and C. Wen, Phys. Rev. Lett. 120, no. 26, 261602 (2018) [arXiv:1801.01496 [hep-th]].

[17] H. Elvang, M. Hadjiantonis, C. R. T. Jones and S. Paranjape, Phys. Lett. B 781, 656 (2018) [arXiv:1712.09937 [hep-th]]. 